\title{
Nonlinear Observer for GNSS-Aided Inertial Navigation with Quaternion-Based Attitude Estimation
}

\author{
Håvard Fjær Grip, Thor I. Fossen, Tor A. Johansen, and Ali Saberi
}

\begin{abstract}
For applications with limited computational capacity, observers designed based on nonlinear stability theory offer an alternative to computationally demanding extended Kalman filters. In this paper, we present a semiglobally stable nonlinear observer for estimating position, velocity, attitude, and gyro bias by combining a GNSS receiver with an inertial measurement unit including a magnetometer. Previous work by the authors on this topic was based on local navigation equations that ignored the Earth's rotation and curvature. Moreover, the attitude was represented by an over-parameterized 9degrees-of-freedom matrix. The current paper improves on these aspects by using navigation equations that take the Earth's rotation and curvature into account, and by representing the attitude estimate as a unit quaternion. Furthermore, the observer is tested experimentally on data from a light fixedwing aircraft.
\end{abstract}

\section{INTRODUCTION}

Strapdown inertial navigation is a method of estimating position, velocity, and attitude (PVA) by integrating accelerometer and rate gyro measurements in a dead-reckoning fashion. Inertial navigation systems (INS) can be highly accurate in the short term, but errors accumulate over time, leading to poor long-term accuracy. For this reason, inertial navigation systems are often aided by absolute reference information, obtained regularly or intermittently from other sources, to help correct the INS estimates and determine sources of error, such as sensor bias (see, e.g., [1], [2]).

Aided INS has traditionally been limited to high-cost applications, due in part to the need for accurate inertial sensors capable of coasting through long periods of time without reference information. With the advent of MEMS technology, however, cheap inertial sensors are appearing in an increasingly wide range of products, such as cars, mobile phones, and game controllers. Although the accuracy of these sensors is limited, they often appear in combination with GNSS receivers, such as GPS, which provide frequent reference information in the form of position fixes and (in many cases) Doppler-based velocity. This development holds the promise of making GNSS-aided inertial navigation widely available at low cost.

Håvard Fjær Grip is affiliated with the Department of Engineering Cybernetics, Norwegian University of Science and Technology, 7491 Trondheim, Norway.

Thor I. Fossen and Tor A. Johansen are affiliated with the Centre for Autonomous Marine Operations and Systems, Department of Engineering Cybernetics, Norwegian University of Science and Technology, 7491 Trondheim, Norway.

Ali Saberi is affiliated with the School of Electrical Engineering and Computer Science, Washington State University, Pullman, WA 99164, USA.

The work of Håvard Fjær Grip was supported by the Research Council of Norway. The work of Ali Saberi was partially supported by NAVY grants ONR KKK777SB001 and ONR KKK760SB0012.
The topic of GNSS/INS integration has been studied for decades, and it is typically accomplished using an extended Kalman filter (EKF) (see, e.g., [3]-[5]). The EKF may not be the best choice for low-cost applications, however, due to its relatively large computational footprint. An alternative approach is to construct lower-order observers based on nonlinear stability theory.

\section{A. Nonlinear Observers for GNSS/INS Integration}

A challenging part of the GNSS/INS integration task is the estimation of attitude. Nonlinear attitude estimation has received significant attention as a stand-alone problem [6][13], producing a number of different designs that rely on either an attitude measurement or a set of vector measurements in the body-fixed frame that can be compared with reference vectors in the navigation frame. An extensive survey of attitude estimation methods is given by Crassidis, Markley, and Cheng [14].

Vik and Fossen [15] expanded the attitude observer of Salcudean [6] to include gyro bias estimation, and used this observer as part of a complete GNSS/INS integration observer. However, their approach assumes the availability of an external attitude measurement, allowing the attitude observer to be implemented as a stand-alone module independent from the position and velocity estimation. In most cases, an attitude measurement is not available, and the attitude estimation must be combined with position and velocity in a more complicated way.

Hua [16] used the vector-based attitude observer of Hamel and Mahony [17] and Mahony, Hamel, and Pflimlin [8] as the basis for an attitude and velocity observer that depends only on inertial measurements, GNSS velocity measurements, and magnetometer measurements. Hua's observer exploits the fact that the vehicle's acceleration vector in the navigation frame is implicitly available in the derivative of the GNSS velocity. This vector can be compared to the accelerometer measurement in order to determine the attitude.

In a similar vein, GNSS/INS integration was used as an application example in the authors' study of observer design for general cascades of nonlinear and linear time-invariant systems [18], [19]. In this case, the attitude estimate was allowed to develop in 9 degrees of freedom (DOF), similar to the work of Batista et al. [10], [11], resulting in a global exponential stability result. This approach has recently been developed into a more complete integration scheme including estimation of gyro bias [20].

The current paper offers three main contributions relative to the authors' previous work: 
Quaternion-based attitude estimation with bias compensation: The attitude observer of Hamel and Mahony [17] and Mahony, Hamel, and Pflimlin [8] includes estimation of gyro bias for the case of stationary reference vectors. The authors recently extended the analysis of this observer by developing a strong Lyapunov function showing semiglobal stability for the case of non-stationary reference vectors as well [12], [13]. We take advantage of this analysis to create a GNSS/INS integration scheme with gyro bias estimation, where the attitude is represented as a unit quaternion instead of a 9-DOF estimate of the rotation matrix.

Earth rotation and curvature: Our previous work was based on local navigation equations that ignored the rotation and curvature of the Earth, as well as the gravity vector's dependence on the vehicle's position. This simplification resulted in a dynamical system that could be viewed as the cascade of a nonlinear and a linear time-invariant system, for which the authors have developed a general $\mathscr{H}_{\infty}$-based theory [18], [19]. Here, we dispense with this simplification and use proper equations that take these factors into account. As a consequence, we need to modify our methodology for ensuring stability of the overall error dynamics.

Experimental testing: Rather than relying on simulations, we test our observer using data from a light fixedwing aircraft. Specifically, we implement the observer based on measurements from a lower-quality inertial measurement unit (IMU) and a GNSS receiver without velocity information, and compare the results to a higher-quality reference.

\section{B. Notation and Preliminaries}

For a vector or matrix $X, X^{\prime}$ denotes its transpose. The operator $\|\cdot\|$ denotes the Euclidean norm for vectors and the induced Euclidean norm for matrices. For a vector $x \in \mathbb{R}^{3}$, $S(x)$ denotes the skew-symmetric matrix

$$
S(x)=\left[\begin{array}{ccc}
0 & -x_{3} & x_{2} \\
x_{3} & 0 & -x_{1} \\
-x_{2} & x_{1} & 0
\end{array}\right] .
$$

For a symmetric positive-definite matrix $P, \lambda_{\min }(P)$ and $\lambda_{\max }(P)$ denote the minimum and maximum eigenvalue, respectively. For a vector $x$, we denote by $\operatorname{sat}_{L}(x)$ an elementwise saturation of $x$ with limits $\pm L$. We denote by $\left[z_{1} ; \ldots ; z_{n}\right]$ the vector obtained by stacking the vectors $z_{1}, \ldots, z_{n}$. All dynamical systems are assumed to be initialized at time $t=0$. For notational simplicity, we shall omit function arguments whenever possible.

A quaternion $q=\left[s_{q} ; r_{q}\right]$ consists of a real part $s_{q} \in \mathbb{R}$ and a vector part $r_{q} \in \mathbb{R}^{3}$. The quaternion product between $q$ and $p$ is given by

$$
q \otimes p=\left[\begin{array}{c}
s_{q} s_{p}-r_{q}^{\prime} r_{p} \\
s_{q} r_{p}+s_{p} r_{q}+r_{q} \times r_{p}
\end{array}\right] .
$$

The conjugate of $q$, given by $\left[s_{q} ;-r_{q}\right]$, is denoted by $q^{*}$. For a vector $x \in \mathbb{R}^{3}$, we denote by $\bar{x}$ the quaternion with real part zero and vector part $x$ (i.e., $\bar{x}=[0 ; x]$ ).

Throughout the paper, we will operate with several different coordinate systems. Unless clear from the context, we use a superscript index to indicate the coordinate system in which a given vector is decomposed; thus, $x^{\mathrm{a}}$ and $x^{\mathrm{b}}$ refer to the same vector decomposed in the coordinate systems indexed by a and $b$, respectively. The rotation between these coordinate systems can be represented by a unit quaternion $q_{\mathrm{a}}^{\mathrm{b}}=\left[s_{q_{\mathrm{a}}^{\mathrm{b}}} ; r_{q_{\mathrm{a}}^{\mathrm{b}}}\right]$, such that $\bar{x}^{\mathrm{b}}=q_{\mathrm{a}}^{\mathrm{b}} \otimes \bar{x}^{\mathrm{a}} \otimes q_{\mathrm{a}}^{\mathrm{b} *}$. The corresponding rotation matrix $R\left(q_{\mathrm{a}}^{\mathrm{b}}\right) \in \mathrm{SO}(3)$ such that $R\left(q_{\mathrm{a}}^{\mathrm{b}}\right) x^{\mathrm{a}}=x^{\mathrm{b}}$ is given as $R\left(q_{\mathrm{a}}^{\mathrm{b}}\right)=I+2 s_{q_{\mathrm{a}}^{\mathrm{b}}} S\left(r_{q_{\mathrm{a}}^{\mathrm{b}}}\right)+2 S\left(r_{q_{\mathrm{a}}^{\mathrm{b}}}\right)^{2}$. The rate of rotation of the coordinate system indexed by $\mathrm{b}$ with respect to $\mathrm{a}$, decomposed in c, is denoted by $\omega_{\mathrm{ab}}^{\mathrm{c}}$.

We shall refer to four coordinate systems in particular, namely, the Earth-Centered Inertial (ECI), Earth-Centered Earth-Fixed (ECEF), North-East-Down (NED), and BodyFixed (BODY) coordinate systems, with corresponding indices i, e, n, and b. For details we refer to Fossen [1].

\section{PRoblem Formulation}

We are interested in estimating PVA by integrating measurements from a GNSS receiver and an IMU including a magnetometer. Because the estimation of attitude is sensitive to bias in the gyro measurements, we shall also estimate the gyro bias $b^{\mathrm{b}}$, which is presumed to be constant. There are several different options for how to represent PVA. Here, we shall represent the position and velocity as vectors $p^{\mathrm{e}}$ and $v^{\mathrm{e}}$ in the ECEF coordinate system. ${ }^{1}$ This representation is convenient because $p^{\mathrm{e}}$ and $v^{\mathrm{e}}$ are quantities typically output by a GNSS receiver. The attitude will be represented as a unit quaternion $q_{\mathrm{b}}^{\mathrm{e}}$ representing the rotation from BODY to ECEF. These representations give rise to the following dynamic equations (see, e.g., [2]):

$$
\begin{aligned}
& \dot{p}^{\mathrm{e}}=v^{\mathrm{e}} \\
& \dot{v}^{\mathrm{e}}=-2 S\left(\omega_{\mathrm{ie}}^{\mathrm{e}}\right) v^{\mathrm{e}}+f^{\mathrm{e}}+g^{\mathrm{e}}\left(p^{\mathrm{e}}\right), \\
& \dot{q}_{\mathrm{b}}^{\mathrm{e}}=\frac{1}{2} q_{\mathrm{b}}^{\mathrm{e}} \otimes \bar{\omega}_{\mathrm{ib}}^{\mathrm{b}}-\frac{1}{2} \bar{\omega}_{\mathrm{ie}}^{\mathrm{e}} \otimes q_{\mathrm{b}}^{\mathrm{e}} \\
& \dot{b}^{\mathrm{b}}=0
\end{aligned}
$$

where $f^{\mathrm{e}}$ denotes the specific force acting on the vehicle and $g^{\mathrm{e}}\left(p^{\mathrm{e}}\right)$ denotes the plumb bob gravity vector, which is a function of the vehicle's position. The known vector $\omega_{\mathrm{ie}}^{\mathrm{e}}$ represents the Earth's rate of rotation around the ECEF $z$-axis.

Remark 1: Our chosen representation of PVA is not necessarily the most practical when it comes to using the estimates for control or for displaying them to a user. However, it is straightforward to transform these quantities to a more practical format, as discussed in Section V.

Remark 2: It may be reasonably argued that the Earth's rate of rotation $\omega_{\mathrm{ie}}^{\mathrm{b}}$ is irrelevant when targeting low-cost applications without highly accurate sensors. Nevertheless, some MEMS-based inertial sensors are already capable of measuring the Earth's rate of rotation and may one day offer a cheap alternative even for highly accurate navigation.

\footnotetext{
${ }^{1}$ As is common, the velocity $v^{\mathrm{e}}$ is here taken to represent the rate of change of the position $p^{\mathrm{e}}$ (that is, $v^{\mathrm{e}}:=\dot{p}^{\mathrm{e}}$ ).
} 


\section{A. Sensor Configuration}

We assume that the following information is available:

- a measurement $p_{\mathrm{GNSS}}^{\mathrm{e}}=p^{\mathrm{e}}$ of the position (from the GNSS receiver)

- a full or partial measurement $v_{\mathrm{GNSS}}^{\mathrm{e}}=C_{v} v^{\mathrm{e}}$ of the velocity (from the GNSS receiver), where the measurement matrix $C_{v}$ may be an empty matrix, the identity matrix, or some other matrix of appropriate dimensions

- a measurement $f_{\mathrm{IMU}}^{\mathrm{b}}=f^{\mathrm{b}}$ of the specific force acting on the vehicle (from the IMU's accelerometers)

- a biased measurement $\omega_{\mathrm{ib}, \mathrm{IMU}}^{\mathrm{b}}=\omega_{\mathrm{ib}}^{\mathrm{b}}+b^{\mathrm{b}}$ of the vehicle's angular velocity (from the IMU's gyros)

- a measurement $m_{\mathrm{IMU}}^{\mathrm{b}}=m^{\mathrm{b}}$ of the Earth's magnetic field (from the IMU's magnetometers)

\section{B. Assumptions}

We assume knowledge of a bound $M_{f}$ on the magnitude of the specific force $f^{\mathrm{b}}$ and a bound $M_{b}$ on the magnitude of the gyro bias. Furthermore, we assume that the angular velocity $\omega_{\mathrm{ib}}^{\mathrm{b}}$ and the time derivative $\dot{f}^{\mathrm{b}}$ of the specific force are uniformly bounded. In order to ensure uniform observability, we also assume that there exists a constant $c_{\mathrm{obs}}>0$ such that $\left\|f^{\mathrm{b}} \times m^{\mathrm{b}}\right\| \geq c_{\text {obs }}$ for all $t \geq 0$. $^{2}$ Finally, we assume that the gravity $g^{\mathrm{e}}\left(p^{\mathrm{e}}\right)$ is a Lipschitz continuous function of the position $p^{\mathrm{e}}$.

\section{AtTitude Estimation}

As a step toward the complete design, we focus first on the subproblem of estimating the attitude $q_{\mathrm{b}}^{\mathrm{e}}$ and gyro bias $b^{\mathrm{b}}$. Throughout this section we shall assume, hypothetically, that we have access to a pair of vector measurements $v_{1}^{\mathrm{b}}$ and $v_{2}^{\mathrm{b}}$ in BODY and a corresponding pair of reference vectors $v_{1}^{\mathrm{e}}$ and $v_{2}^{\mathrm{e}}$ in ECEF, with the property that $\left\|v_{1}^{\mathrm{b}} \times v_{2}^{\mathrm{b}}\right\| \geq c_{\text {obs }}>0$. Based on this type of vector information, Hamel and Mahony [17] and Mahony, Hamel, and Pflimlin [8] presented an elegant solution that guarantees almost-global asymptotic stability for the case of constant reference vectors. The stability analysis was recently extended by the authors to prove semiglobal stability in the case of time-varying reference vectors [12], [13]. Modified to account for the rotation of the Earth, the observer equations can be written as follows:

$$
\begin{aligned}
& \dot{\hat{q}}_{\mathrm{b}}^{\mathrm{e}}=\frac{1}{2} \hat{q}_{\mathrm{b}}^{\mathrm{e}} \otimes\left(\bar{\omega}_{\mathrm{ib}, I M U}^{\mathrm{b}}-\overline{\hat{b}}^{\mathrm{b}}+\bar{\sigma}\right)-\frac{1}{2} \bar{\omega}_{\mathrm{ie}}^{\mathrm{e}} \otimes \hat{q}_{\mathrm{b}}^{\mathrm{e}}, \\
& \dot{\hat{b}}^{\mathrm{e}}=\operatorname{Proj}\left(\hat{b}^{\mathrm{b}},-k_{I} \sigma\right),
\end{aligned}
$$

where $k_{I}>0$ is a gain, $\sigma$ is an injection term, and $\operatorname{Proj}(\cdot, \cdot)$ denotes a parameter projection that restricts the parameter estimate $\hat{b}^{\mathrm{b}}$ to the compact set defined by $\left\|\hat{b}^{\mathrm{b}}\right\| \leq M_{\hat{b}}$, where $M_{\hat{b}}>M_{b}$ (see [13], [20] for details). ${ }^{3}$ The injection term is defined as

$$
\sigma:=k_{1} v_{1}^{\mathrm{b}} \times R\left(\hat{q}_{\mathrm{b}}^{\mathrm{e}}\right)^{\prime} v_{1}^{\mathrm{e}}+k_{2} v_{2}^{\mathrm{b}} \times R\left(\hat{q}_{\mathrm{b}}^{\mathrm{e}}\right)^{\prime} v_{2}^{\mathrm{e}},
$$

\footnotetext{
${ }^{2}$ Although this assumption is needed in the analysis, the observer equations are well-defined even when it does not hold, and brief violations are of little practical consequence.

${ }^{3}$ Recall that, according to the notation introduced in Section I-B, $\bar{\sigma}=$ $[0 ; \sigma]$ and $\overline{\hat{b}}^{\mathrm{b}}=\left[0 ; \hat{b}^{\mathrm{b}}\right]$.
}

where $k_{1}$ and $k_{2}$ are gains satisfying $k_{1} \geq k_{P}$ and $k_{2} \geq k_{P}$ for some $k_{P}>0$.

To state a stability result for this observer, define the estimation errors $\tilde{q}:=q_{\mathrm{b}}^{\mathrm{e}} \otimes \hat{q}_{\mathrm{b}}^{\mathrm{e} *}$ and $\tilde{b}:=b^{\mathrm{b}}-\hat{b}^{\mathrm{b}}$, and let $\tilde{s}$ and $\tilde{r}$ denote the real part and the vector part of $\tilde{q}$, respectively. Note that $\tilde{r}=0$ (equivalently $|\tilde{s}|=1$ ) corresponds to zero attitude error, whereas $\tilde{s}=0$ corresponds to the maximum attitude error of $180^{\circ}$ around some axis. We shall show that a combined error variable $\tilde{\chi}:=[\tilde{r} ; \tilde{b}]$ converges exponentially to zero from a set of initial conditions that can be made arbitrarily large.

Let $\tilde{\mathscr{Q}}(\varepsilon)=\{\tilde{q}|| \tilde{s} \mid>\varepsilon\}$ represent a set of attitude errors bounded away from $180^{\circ}$ by a margin determined by $\varepsilon \in$ $\left(0, \frac{1}{2}\right)$. Then we have the following semiglobal exponential stability result:

Lemma 1: For each $\varepsilon \in\left(0, \frac{1}{2}\right)$, there exists a $k_{P}^{*}>0$ such that, if $k_{P}>k_{P}^{*}$, then for all initial conditions such that $\tilde{q}(0) \in$ $\tilde{\mathscr{Q}}(\varepsilon)$ and $\left\|\hat{b}^{\mathrm{b}}(0)\right\| \leq M_{\hat{b}}$,

$$
\|\tilde{\chi}(t)\| \leq K \mathrm{e}^{-\lambda t}\|\tilde{\chi}(0)\|,
$$

for some constants $K>0$ and $\lambda>0$.

Proof: The proof is only a slight extension of the authors' previous work [13, Theorem 1], and we only outline the necessary changes here. It is straightforward to confirm that

$$
\begin{aligned}
& \dot{\tilde{s}}=\frac{1}{2} \tilde{r}^{\prime} R\left(q_{\mathrm{b}}^{\mathrm{e}}\right)(\tilde{b}+\sigma), \\
& \dot{\tilde{r}}=-\frac{1}{2}(I \tilde{s}-S(\tilde{r})) R\left(q_{\mathrm{b}}^{\mathrm{e}}\right)(\tilde{b}+\sigma)+S(\tilde{r}) \omega_{\mathrm{ie}}^{\mathrm{e}}, \\
& \dot{\tilde{b}}=-\operatorname{Proj}\left(\hat{b}^{\mathrm{b}},-k_{I} \sigma\right),
\end{aligned}
$$

which differs from [13] only in the term $S(\tilde{r}) \omega_{\mathrm{ie}}^{e}$. The parameter projection ensures that $\|\tilde{b}\| \leq M$, where $M>0$ is from [13]. Considering the function $V(\tilde{s})=1-\tilde{s}^{2}=\|\tilde{r}\|^{2}$ from [13], we therefore have $\dot{V} \leq M-k_{P} c_{\text {obs }}^{2} \alpha(\tilde{s})$, where $\alpha(\tilde{s}):=$ $\tilde{s}^{2}\left(1-\tilde{s}^{2}\right)$. Hence, for sufficiently large $k_{P},|\tilde{s}|=\varepsilon \Longrightarrow$ $\dot{V} \leq M-k_{P} c_{\mathrm{obs}}^{2} \alpha(\varepsilon)<0$, which implies that the trajectories cannot escape the region defined by $|\tilde{s}| \geq \varepsilon$. Considering next the function $W(t, \tilde{r}, \tilde{s}, \tilde{b})=V(\tilde{s})+2 \ell \tilde{s} \tilde{r}^{\prime} R\left(q_{\mathrm{b}}^{\mathrm{e}}\right) \tilde{b}+\frac{\ell}{2 k_{I}} \tilde{b}^{\prime} \tilde{b}$ from [13], we get

$$
\dot{W}=f_{W}(t, \tilde{s}, \tilde{r}, \tilde{b})-2 \ell \tilde{s} \omega_{\mathrm{ie}}^{\mathrm{e}}{ }^{\prime} S(\tilde{r}) R\left(q_{\mathrm{b}}^{\mathrm{e}}\right) \tilde{b},
$$

where $f_{W}(t, \tilde{s}, \tilde{r}, \tilde{b})$ is the derivative from [13] with $\omega=\omega_{\mathrm{eb}}^{\mathrm{b}}$. Noting that $2 \ell \tilde{s} \omega_{\mathrm{ie}}^{\mathrm{e}} ' S(\tilde{r}) R\left(q_{\mathrm{b}}^{\mathrm{e}}\right) \tilde{b} \leq 2 \ell\left\|\omega_{\mathrm{ie}}^{\mathrm{e}}\right\|\|\tilde{r}\|\|\tilde{b}\|$ and using the results from [13], we can therefore write

$$
\dot{W} \leq-\left[\begin{array}{ll}
\|\tilde{r}\| & \|\tilde{b}\|
\end{array}\right]\left[\begin{array}{cc}
k_{P} a-\ell M^{2} & \star \\
-\frac{1}{2}\left(1+2 \ell\left(M_{\omega}+\left\|\omega_{\mathrm{ie}}^{\mathrm{e}}\right\|\right)\right) & -\ell \varepsilon^{2}
\end{array}\right]\left[\begin{array}{l}
\|\tilde{r}\| \\
\|\tilde{b}\|
\end{array}\right],
$$

where $a>0$ is from [13] and $M_{\omega} \geq\left\|\omega_{\mathrm{eb}}^{\mathrm{b}}\right\|$, and where $\star$ is used to indicate matrix symmetry. Using the same techniques as in [13], we can now prove that for sufficiently large $k_{P}$, $\dot{W} \leq-\kappa\|\tilde{\chi}\|^{2}$ for some $\kappa>0$, and the result follows from the comparison lemma [21, Lemma 3.4]. 


\section{NONLINEAR INTEGRATION OBSERVER}

In this section we shall employ the attitude and gyro bias observer as part of a GNSS/INS integration observer based on the available measurements. To this end, we need a pair of vector measurements $v_{1}^{\mathrm{b}}$ and $v_{2}^{\mathrm{b}}$ in the BODY frame and a corresponding pair of reference vectors $v_{1}^{\mathrm{e}}$ and $v_{2}^{\mathrm{e}}$ in the ECEF frame, as indicated in the last section.

We use the accelerometer measurement $f^{\mathrm{b}}$ and the magnetometer measurement $m^{\mathrm{b}}$ to generate two vectors in the BODY frame. There are several ways to do this, as discussed in Section V; for ease of presentation, however, we shall simply use $v_{1}^{\mathrm{b}}:=m^{\mathrm{b}}$ and $v_{2}^{\mathrm{b}}:=f^{\mathrm{b}}$ in this section, and note that $\left\|v_{1}^{\mathrm{b}} \times v_{2}^{\mathrm{b}}\right\| \geq c_{\mathrm{obs}}$ is then satisfied by assumption. We can assume that the reference vector $v_{1}^{\mathrm{e}}=m^{\mathrm{e}}$ (that is, the Earth's magnetic field vector decomposed in ECEF) is known. However, the reference vector $v_{2}^{\mathrm{e}}=f^{\mathrm{e}}$ (that is, the specific force decomposed in ECEF) is not measured. To deal with this problem, we shall substitute $v_{2}^{\mathrm{e}}$ in the observer with $\hat{v}_{2}^{\mathrm{e}}:=\operatorname{sat}_{M_{f}}\left(\hat{f}^{\mathrm{e}}\right)$, where $\hat{f}^{\mathrm{e}}$ is an estimate of $f^{\mathrm{e}}$. Consequently, the attitude and gyro bias observer is now given by

$$
\begin{aligned}
& \dot{\hat{q}}_{\mathrm{b}}^{\mathrm{e}}=\frac{1}{2} \hat{q}_{\mathrm{b}}^{\mathrm{e}} \otimes\left(\bar{\omega}_{\mathrm{ib}, \mathrm{IMU}}^{\mathrm{b}}-\overline{\hat{b}}^{\mathrm{b}}+\overline{\hat{\sigma}}\right)-\frac{1}{2} \bar{\omega}_{\mathrm{ie}}^{\mathrm{e}} \otimes \hat{q}_{\mathrm{b}}^{\mathrm{e}}, \\
& \hat{b}^{\mathrm{b}}=\operatorname{Proj}\left(\hat{b}^{\mathrm{b}},-k_{I} \hat{\sigma}\right),
\end{aligned}
$$

where

$$
\hat{\sigma}:=k_{1} m_{\mathrm{IMU}}^{\mathrm{b}} \times R\left(\hat{q}_{\mathrm{b}}^{\mathrm{e}}\right)^{\prime} m^{\mathrm{e}}+k_{2} f_{\mathrm{IMU}}^{\mathrm{b}} \times R\left(\hat{q}_{\mathrm{b}}^{\mathrm{e}}\right)^{\prime} \operatorname{sat}_{M_{f}}\left(\hat{f}^{\mathrm{e}}\right) .
$$

Compared to the observer in Section III, the injection term $\sigma$ has been replaced by $\hat{\sigma}$.

The estimate $\hat{f}^{\mathrm{e}}$ is generated together with the position estimate $\hat{p}^{\mathrm{e}}$ and the velocity estimate $\hat{v}^{\mathrm{e}}$ by the following observer:

$$
\begin{aligned}
\dot{\hat{p}}^{\mathrm{e}}= & \hat{v}^{\mathrm{e}}+\theta K_{p p}\left(p_{\mathrm{GNSS}}^{\mathrm{e}}-\hat{p}^{\mathrm{e}}\right)+K_{p v}\left(v_{\mathrm{GNSS}}^{\mathrm{e}}-C_{v} \hat{v}^{\mathrm{e}}\right), \\
\hat{\hat{v}}^{\mathrm{e}}= & -2 S\left(\omega_{\mathrm{ie}}^{\mathrm{e}}\right) \hat{v}^{\mathrm{e}}+\hat{f}^{\mathrm{e}}+g^{\mathrm{e}}\left(\hat{p}^{\mathrm{e}}\right) \\
& +\theta^{2} K_{v p}\left(p_{\mathrm{GNSS}}^{\mathrm{e}}-\hat{p}^{\mathrm{e}}\right)+\theta K_{v v}\left(v_{\mathrm{GNSS}}^{\mathrm{e}}-C_{v} \hat{v}^{\mathrm{e}}\right), \\
\dot{\xi}= & -R\left(\hat{q}_{\mathrm{b}}^{\mathrm{e}}\right) S(\hat{\sigma}) f_{\mathrm{IMU}}^{\mathrm{b}} \\
& +\theta^{3} K_{\xi_{p}}\left(p_{\mathrm{GNSS}}^{\mathrm{e}}-\hat{p}^{\mathrm{e}}\right)+\theta^{2} K_{\xi v}\left(v_{\mathrm{GNSS}}^{\mathrm{e}}-C_{v} \hat{v}^{\mathrm{e}}\right), \\
\hat{f}^{\mathrm{e}}= & R\left(\hat{q}_{\mathrm{b}}^{\mathrm{e}}\right) f_{\mathrm{IMU}}^{\mathrm{b}}+\xi
\end{aligned}
$$

where $K_{p p}, K_{p v}, K_{v p}, K_{v v}, K_{\xi p}$, and $K_{\xi v}$ are gain matrices. These gain matrices are chosen freely to ensure that the matrix $A-K C$ is Hurwitz, where

$$
A=\left[\begin{array}{ccc}
0 & I_{3} & 0 \\
0 & 0 & I_{3} \\
0 & 0 & 0
\end{array}\right], C=\left[\begin{array}{ccc}
I_{3} & 0 & 0 \\
0 & C_{v} & 0
\end{array}\right], K=\left[\begin{array}{cc}
K_{p p} & K_{p v} \\
K_{v p} & K_{v v} \\
K_{\xi p} & K_{\xi c}
\end{array}\right] .
$$

The parameter $\theta \geq 1$ will be used as a tuning parameter to guarantee stability.

To state our main result, define the estimation errors $\tilde{p}:=$ $p^{\mathrm{e}}-\hat{p}^{\mathrm{e}}, \tilde{v}:=v^{\mathrm{e}}-\hat{v}^{\mathrm{e}}$, and $\tilde{f}:=f^{\mathrm{e}}-\hat{f}^{\mathrm{e}}$, and the combined error vector $\tilde{x}:=[\tilde{p} ; \tilde{v} ; \tilde{f}]$. Our main semiglobal stability result can now be stated as follows.

Theorem 1: Let $\mathscr{K} \in \mathbb{R}^{9}$ be an arbitrary compact set containing the origin, and let $\bar{\varepsilon} \in\left(0, \frac{1}{2}\right)$ be an arbitrary constant. Let $k_{P}$ be chosen to ensure stability according to
Lemma 1 with respect to some $\varepsilon<\bar{\varepsilon}$. There exists a $\theta^{*} \geq 1$ such that, if $\theta \geq \theta^{*}$, then for all initial conditions such that $(\tilde{p}(0) \times \tilde{v}(0) \times \overline{\tilde{\xi}}(0)) \in \mathscr{K}, \tilde{q}(0) \in \tilde{\mathscr{Q}}(\bar{\varepsilon})$, and $\left\|\hat{b}^{\mathrm{b}}(0)\right\| \leq M_{\hat{b}}$,

$$
\sqrt{\|\tilde{x}(t)\|^{2}+\|\tilde{\chi}(t)\|^{2}} \leq K \mathrm{e}^{-\lambda t} \sqrt{\|\tilde{x}(0)\|^{2}+\|\tilde{\chi}(0)\|^{2}},
$$

for some $K>0$ and $\lambda>0$.

Proof: The dynamics of $\tilde{p}$ and $\tilde{v}$ is given by

$$
\begin{aligned}
\dot{\tilde{p}} & =\tilde{v}-\theta K_{p p} \tilde{p}-K_{p v} C_{v} \tilde{v} \\
\dot{\tilde{v}} & =-2 S\left(\omega_{\mathrm{ie}}^{\mathrm{e}}\right) \tilde{v}+\tilde{f}+g^{\mathrm{e}}\left(p^{\mathrm{e}}\right)-g^{\mathrm{e}}\left(\hat{p}^{\mathrm{e}}\right)-\theta^{2} K_{v p} \tilde{p}-\theta K_{v v} C_{v} \tilde{v} .
\end{aligned}
$$

Noting that $\dot{R}\left(q_{\mathrm{b}}^{\mathrm{e}}\right)=R\left(q_{\mathrm{b}}^{\mathrm{e}}\right) S\left(\omega_{\mathrm{ib}}^{\mathrm{b}}\right)-S\left(\omega_{\mathrm{ie}}^{\mathrm{e}}\right) R\left(q_{\mathrm{b}}^{\mathrm{e}}\right)$ and $\dot{R}\left(\hat{q}_{\mathrm{b}}^{\mathrm{e}}\right)=$ $R\left(\hat{q}_{\mathrm{b}}^{\mathrm{e}}\right) S\left(\omega_{\mathrm{ib}}^{\mathrm{b}}+\tilde{b}+\hat{\sigma}\right)-S\left(\omega_{\mathrm{ie}}^{\mathrm{e}}\right) R\left(\hat{q}_{\mathrm{b}}^{\mathrm{e}}\right)$, and defining $\tilde{R}:=$ $R\left(q_{\mathrm{b}}^{\mathrm{e}}\right) R\left(\hat{q}_{\mathrm{b}}^{\mathrm{e} *}\right)=R(\tilde{q})$, the dynamics of $\tilde{f}=f^{\mathrm{e}}-\hat{f}^{\mathrm{e}}=$ $R\left(q_{\mathrm{b}}^{\mathrm{e}}\right) f^{\mathrm{b}}-R\left(\hat{q}_{\mathrm{b}}^{\mathrm{e}}\right) f^{\mathrm{b}}-\xi$ can be calculated as

$$
\begin{aligned}
\dot{\tilde{f}}= & \dot{R}\left(q_{\mathrm{b}}^{\mathrm{e}}\right) f^{\mathrm{b}}+R\left(q_{\mathrm{b}}^{\mathrm{e}}\right) \dot{f}^{\mathrm{b}}-\dot{R}\left(\hat{q}_{\mathrm{b}}^{\mathrm{e}}\right) f^{\mathrm{b}}-R\left(\hat{q}_{\mathrm{b}}^{\mathrm{e}}\right) \dot{f}^{\mathrm{b}}-\dot{\xi} \\
= & R\left(q_{\mathrm{b}}^{\mathrm{e}}\right) S\left(\omega_{\mathrm{ib}}^{\mathrm{b}}\right) f^{\mathrm{b}}-S\left(\omega_{\mathrm{ie}}^{\mathrm{e}}\right) R\left(q_{\mathrm{b}}^{\mathrm{e}}\right) f^{\mathrm{b}}+R\left(q_{\mathrm{b}}^{\mathrm{e}}\right) \dot{f}^{\mathrm{b}} \\
& -R\left(\hat{q}_{\mathrm{b}}^{\mathrm{e}}\right) S\left(\omega_{\mathrm{ib}}^{\mathrm{b}}+\tilde{b}+\hat{\sigma}\right) f^{\mathrm{b}}+S\left(\omega_{\mathrm{ie}}^{\mathrm{e}}\right) R\left(\hat{q}_{\mathrm{b}}^{\mathrm{e}}\right) f^{\mathrm{b}}-R\left(\hat{q}_{\mathrm{b}}^{\mathrm{e}}\right) \dot{f}^{\mathrm{b}} \\
& +R\left(\hat{q}_{\mathrm{b}}^{\mathrm{e}}\right) S(\hat{\sigma}) f^{\mathrm{b}}-\theta^{3} K_{\xi p} \tilde{p}-\theta^{2} K_{\xi_{v}} C_{v} \tilde{v} \\
= & \tilde{d}-\theta^{3} K_{\xi p} \tilde{p}-\theta^{2} K_{\xi \nu} C_{v} \tilde{v},
\end{aligned}
$$

where

$$
\begin{aligned}
\tilde{d}= & \left(I-\tilde{R}^{\prime}\right) R\left(q_{\mathrm{b}}^{\mathrm{e}}\right)\left(S\left(\omega_{\mathrm{ib}}^{\mathrm{b}}\right) f^{\mathrm{b}}+\dot{f}^{\mathrm{b}}\right) \\
& -S\left(\omega_{\mathrm{ie}}^{\mathrm{e}}\right)\left(I-\tilde{R}^{\prime}\right) R\left(q_{\mathrm{b}}^{\mathrm{e}}\right) f^{\mathrm{b}}-\tilde{R}^{\prime} R\left(q_{\mathrm{b}}^{\mathrm{e}}\right) S(\tilde{b}) f^{\mathrm{b}} .
\end{aligned}
$$

Next, let $\eta_{1}:=\tilde{p}, \quad \eta_{2}:=\tilde{v} / \theta, \quad \eta_{3}:=\tilde{f} / \theta^{2}$, and $\eta:=$ $\left[\eta_{1} ; \eta_{2} ; \eta_{3}\right]$. Then it is easy to confirm that

$$
\frac{1}{\theta} \dot{\eta}=(A-K C) \eta+\rho_{1}(t, \eta)+\rho_{2}(t, \tilde{\chi})
$$

where

$$
\rho_{1}(t, \eta)=\left[\begin{array}{c}
0 \\
-\frac{1}{\theta} 2 S\left(\omega_{\mathrm{ie}}^{\mathrm{e}}\right) \eta_{2}+\frac{1}{\theta^{2}}\left(g^{\mathrm{e}}\left(p^{\mathrm{e}}\right)-g^{\mathrm{e}}\left(p^{\mathrm{e}}-\eta_{1}\right)\right) \\
0
\end{array}\right]
$$

and $\rho_{2}(t, \tilde{\chi})=\left[0 ; 0 ; \frac{1}{\theta^{3}} \tilde{d}\right]$. Using the Lipschitz property of $g^{\mathrm{e}}$, it is easy to see that $\left\|\rho_{1}(t, \eta)\right\| \leq \frac{1}{\theta} \gamma_{1}\|\eta\|$ for some $\gamma_{1}>0$ independent of $\theta$. Furthermore, noting that $\left\|I-\tilde{R}^{\prime}\right\|=$ $\left\|\tilde{s} S(\tilde{r})-S(\tilde{r})^{2}\right\| \leq 2\|\tilde{r}\|$, it is easy to confirm that $\left\|\rho_{2}(t, \tilde{\chi})\right\| \leq$ $\frac{1}{\theta^{3}} \gamma_{2}\|\tilde{\chi}\|$ for some $\gamma_{2}>0$ independent of $\theta$.

Let $P=P^{\prime}>0$ be the solution of the Lyapunov equation $P(A-K C)+(A-K C)^{\prime} P=-I$ and define $U=\frac{1}{\theta} \eta^{\prime} P \eta$. Then

$$
\begin{aligned}
\dot{U} & =-\|\eta\|^{2}+2 \eta^{\prime} P\left(\rho_{1}(t, \eta)+\rho_{2}(t, \tilde{\chi})\right) \\
& \leq-\left(1-\frac{2\|P\| \gamma_{1}}{\theta}\right)\|\eta\|^{2}+\frac{2\|P\| \gamma_{2}}{\theta^{3}}\|\eta\|\|\tilde{\chi}\| .
\end{aligned}
$$

Using this function, we can state the following lemma.

Lemma 2: For any $\delta>0$ and $T>0$, there exists a $\theta_{1}^{*} \geq 1$ such that, if $\theta \geq \theta_{1}^{*}$, then for all initial conditions as specified in Theorem 1, we have that for all $t \geq T,\|\tilde{x}\| \leq \delta$.

Proof: Because the parameter projection ensures $\|\tilde{b}\| \leq$ $M$ and because $\|\tilde{r}\| \leq 1$, we have $\|\tilde{\chi}\| \leq \sqrt{M^{2}+1}$. Define the level set $\Omega_{\theta}=\left\{\eta \mid U \leq \frac{\delta^{2}}{\theta^{5}} \lambda_{\min }(P)\right\}$, and note that 
$\eta \in \Omega_{\theta} \Longrightarrow\|\eta\| \leq \frac{\delta}{\theta^{2}} \Longrightarrow\|\tilde{x}\| \leq \delta$. Outside $\Omega_{\theta}$, we have $\|\eta\| \geq \frac{\delta}{\theta^{2}} \sqrt{\lambda_{\min }(P) / \lambda_{\max }(P)}$, which implies

$$
\begin{aligned}
\dot{U}= & -\left(\frac{1}{2}-\frac{2\|P\| \gamma_{1}}{\theta}\right)\|\eta\|^{2} \\
& -\left(\frac{\delta \sqrt{\lambda_{\min }(P)}}{2 \theta^{2} \sqrt{\lambda_{\max }(P)}}-\frac{2\|P\| \gamma_{2}}{\theta^{3}} \sqrt{M^{2}+1}\right)\|\eta\| .
\end{aligned}
$$

For all sufficiently large $\theta$, the first term can be made smaller than $-\frac{1}{4}\|\eta\|^{2}$ and the second term can be made negative, thus yielding $\dot{U} \leq-\frac{1}{4}\|\eta\|^{2} \leq-\frac{\theta}{4 \lambda_{\max }(P)} U$. By the comparison lemma, we therefore have $U(t) \leq$ $U(0) \exp \left(-\theta t /\left(4 \lambda_{\max }(P)\right)\right)$. Let $L>\delta$ be a bound on $\|\tilde{x}(0)\|$ for any initial condition as specified in Theorem 1 . Then $L$ is also a bound on $\|\eta(0)\|$, and we therefore have $U(t) \leq$ $\frac{1}{\theta} \lambda_{\max }(P) L^{2} \exp \left(-\theta t /\left(4 \lambda_{\max }(P)\right)\right)$ outside $\Omega_{\theta}$. This implies that $\eta$ must enter $\Omega_{\theta}$ before the time

$$
\begin{aligned}
t^{*} & =-\frac{4 \lambda_{\max }(P)}{\theta} \ln \left(\frac{\delta^{2} \lambda_{\min }(P)}{\theta^{4} \lambda_{\max }(P) L^{2}}\right) \\
& =\frac{4 \lambda_{\max }(P)}{\theta}\left(4 \ln (\theta)-\ln \left(\frac{\delta^{2} \lambda_{\min }(P)}{\lambda_{\max }(P) L^{2}}\right)\right) .
\end{aligned}
$$

Noting that $\ln (\theta) / \theta \rightarrow 0$ as $\theta \rightarrow \infty$, we see that for all sufficiently large $\theta \geq 1, t^{*} \leq T$.

Next, consider the dynamics of $\tilde{s}$, given by

$$
\dot{\tilde{s}}=\frac{1}{2} \tilde{r}^{\prime} R\left(q_{\mathrm{b}}^{\mathrm{e}}\right)(\tilde{b}+\hat{\sigma})=\frac{1}{2} \tilde{r}^{\prime} R\left(q_{\mathrm{b}}^{\mathrm{e}}\right)(\tilde{b}+\sigma)+\mu_{1},
$$

where $\mu_{1}=\frac{1}{2} \tilde{r}^{\prime} R\left(q_{\mathrm{b}}^{\mathrm{e}}\right)(\hat{\sigma}-\sigma)$ has the property $\left|\mu_{1}\right| \leq$ $\frac{1}{2} k_{2}\left\|f^{\mathrm{b}}\right\|\|\tilde{r}\|\|\tilde{f}\| \leq \gamma_{3}\|\tilde{r}\|\|\tilde{f}\|$ for an appropriate $\gamma_{3}>0$ independent of $\theta$. We also have $|\dot{\tilde{s}}| \leq \frac{1}{2}(\|\tilde{b}\|+\|\hat{\sigma}\|)$. Since $\|\tilde{b}\| \leq M$ and $\hat{\sigma}$ is made up only of bounded signals, we can conclude that $|\dot{\tilde{s}}| \leq M_{s}$ for some $M_{s}>0$ independent of $\theta$.

With reference to Lemma 2 , define $\delta=k_{P} c_{\text {obs }}^{2}(\alpha(\varepsilon+$ $\tilde{\varepsilon} / 2)-\alpha(\varepsilon)) /\left(2 \gamma_{3}\right)>0$ and $T=\tilde{\varepsilon} /\left(2 M_{s}\right)$, where $\tilde{\varepsilon}:=\bar{\varepsilon}-\varepsilon$, and let $\theta$ be large enough that for all $t \geq T,\|\tilde{x}\| \leq \delta$. Then

$$
|\tilde{s}(T)| \geq|\tilde{s}(0)|-\int_{0}^{T}|\dot{\tilde{s}}(t)| \mathrm{d} t \geq \bar{\varepsilon}-M_{s} \tilde{\varepsilon} /\left(2 M_{s}\right)=\varepsilon+\tilde{\varepsilon} / 2,
$$

and for all $t \geq T,\left|\mu_{1}\right| \leq \gamma_{3}\|\tilde{r}\|\|\tilde{f}\| \leq \gamma_{3} \delta \leq k_{P} c_{\text {obs }}^{2}(\alpha(\varepsilon+$ $\tilde{\varepsilon} / 2)-\alpha(\varepsilon)) / 2$. Consider now the derivative of the function $V(\tilde{s})$ from the proof of Lemma 1 for $t \geq T$ :

$$
\begin{aligned}
\dot{V} & \leq M-k_{P} c_{\text {obs }}^{2} \alpha(\tilde{s})+2\left|\tilde{s} \mu_{1}\right| \\
& \leq M-k_{P} c_{\text {obs }}^{2}(\alpha(\tilde{s})-\alpha(\varepsilon+\tilde{\varepsilon} / 2)+\alpha(\varepsilon)) .
\end{aligned}
$$

With reference to the proof of Lemma 1, it follows that for $|\tilde{s}|=\varepsilon+\tilde{\varepsilon} / 2, \dot{V} \leq M-k_{P} c_{\mathrm{obs}}^{2} \alpha(\varepsilon)<0$. Thus, $\tilde{q}$ cannot escape the set $\tilde{\mathscr{Q}}(\varepsilon+\tilde{\varepsilon} / 2) \subset \tilde{\mathscr{Q}}(\varepsilon)$, and we can assume $|\tilde{s}| \geq \varepsilon$ in the remainder of the analysis.

The dynamics of $\tilde{s}$ differ from (5) only by the term $\mu_{1}$, which can also be given the bound $\left|\mu_{1}\right| \leq \theta^{2} \gamma_{3}\|\tilde{r}\|\|\eta\|$. The dynamics of $\tilde{r}$ and $\tilde{b}$ is given by

$$
\begin{aligned}
& \dot{\tilde{r}}=-\frac{1}{2}(I \tilde{s}-S(\tilde{r})) R\left(q_{\mathrm{b}}^{\mathrm{e}}\right)(\tilde{b}+\sigma)+S(\tilde{r}) \omega_{\mathrm{ie}}^{\mathrm{e}}+\mu_{2}, \\
& \dot{\tilde{b}}=-\operatorname{Proj}\left(\hat{b}^{\mathrm{b}},-k_{I} \sigma\right)+\mu_{3},
\end{aligned}
$$

where $\mu_{2}=\frac{1}{2}(I \tilde{s}-S(\tilde{r})) R\left(q_{\mathrm{b}}^{\mathrm{e}}\right)(\sigma-\hat{\sigma})$ and $\mu_{3}=$ $\operatorname{Proj}\left(\hat{b}^{\mathrm{b}},-k_{I} \sigma\right)-\operatorname{Proj}\left(\hat{b}^{\mathrm{b}},-k_{I} \hat{\sigma}\right)$, which differs from (5) only by the terms $\mu_{2}$ and $\mu_{3}$. We have that $\left\|\mu_{2}\right\| \leq \gamma_{4}\|\tilde{f}\| \leq \theta^{2} \gamma_{4}\|\eta\|$ for some $\gamma_{4}>0$ independent of $\theta$. It can also be shown by the properties of the parameter projection that $\mu_{3} \leq \gamma_{5}\|\tilde{f}\| \leq \theta^{2} \gamma_{5}\|\eta\|$ for some $\gamma_{5}>0$ independent of $\theta$. Considering again the function $W$ from the proof of Lemma 1, we get

$$
\begin{aligned}
\dot{W}= & g_{W}(t, \tilde{s}, \tilde{r}, \tilde{b})-2 \tilde{s} \mu_{1}+2 \ell \mu_{1} \tilde{r}^{\prime} R\left(q_{\mathrm{b}}^{\mathrm{e}}\right) \tilde{b}+2 \ell \tilde{s} \mu_{2}^{\prime} R\left(q_{\mathrm{b}}^{\mathrm{e}}\right) \tilde{b} \\
& +2 \ell \tilde{s} \tilde{r}^{\prime} R\left(q_{\mathrm{b}}^{\mathrm{e}}\right) \mu_{3}+\frac{\ell}{k_{I}} \tilde{b}^{\prime} \mu_{3},
\end{aligned}
$$

where $g_{W}(t, \tilde{s}, \tilde{r}, \tilde{b}) \leq-\kappa\|\tilde{\chi}\|^{2}$ is the derivative of $W$ from the proof of Lemma 1 . Using the bounds on $\mu_{1}, \mu_{2}$, and $\mu_{3}$, we therefore have

$$
\begin{aligned}
\dot{W} \leq & -\kappa\|\tilde{\chi}\|^{2}+2 \theta^{2} \gamma_{3}\|\tilde{r}\|\|\eta\|+2 \ell \theta^{2} \gamma_{3}\|\tilde{b}\|\|\eta\| \\
& +2 \ell \theta^{2} \gamma_{4}\|\tilde{b}\|\|\eta\|+2 \ell \theta^{2} \gamma_{5}\|\tilde{r}\|\|\eta\|+\frac{\ell}{k_{I}} \theta^{2} \gamma_{5}\|\tilde{b}\|\|\eta\| \\
\leq & -\kappa\|\tilde{\chi}\|^{2}+\gamma_{6} \theta^{2}\|\tilde{\chi}\|\|\eta\|,
\end{aligned}
$$

for an appropriate constant $\gamma_{6}$, independent of $\theta$.

We now construct a Lyapunov function $Y=U+\frac{1}{\theta^{5}} W$, which has the property $\beta_{1}\left(\|\eta\|^{2}+\|\tilde{\chi}\|^{2}\right) \leq Y \leq \beta_{2}\left(\|\eta\|^{2}+\right.$ $\|\tilde{\chi}\|^{2}$ ) for some $\beta_{1}>0$ and $\beta_{2}>0$. The derivative satisfies

$$
\begin{aligned}
\dot{Y} \leq & -\left(1-\frac{2\|P\| \gamma_{1}}{\theta}\right)\|\eta\|^{2}+\frac{2\|P\| \gamma_{2}}{\theta^{3}}\|\eta\|\|\tilde{\chi}\| \\
& -\frac{1}{\theta^{5}} \kappa\|\tilde{\chi}\|^{2}+\frac{\gamma_{6}}{\theta^{3}}\|\tilde{\chi}\|\|\eta\| .
\end{aligned}
$$

Assuming $\theta$ is chosen large enough that $1-2\|P\| \gamma_{1} / \theta \geq 1 / 2$, we can write

$$
\dot{Y} \leq-\left[\begin{array}{ll}
\|\eta\| & \|\tilde{\chi}\|
\end{array}\right]\left[\begin{array}{cc}
\frac{1}{2} & -\frac{2\|P\| \gamma_{2}+\gamma_{6}}{2 \theta^{3}} \\
-\frac{2\|P\| \gamma_{2}+\gamma_{6}}{2 \theta^{3}} & \frac{\kappa}{\theta^{5}}
\end{array}\right]\left[\begin{array}{l}
\|\eta\| \\
\|\tilde{\chi}\|
\end{array}\right] .
$$

The first-order principal minor of the above matrix is $\frac{1}{2}>0$. The second-order principal minor is

$$
\frac{\kappa}{2 \theta^{5}}-\frac{\left(2\|P\| \gamma_{2}+\gamma_{6}\right)^{2}}{4 \theta^{6}}
$$

which is positive if $\theta>\left(2\|P\| \gamma_{2}+\gamma_{6}\right)^{2} /(2 \kappa)$. Hence, $\dot{Y} \leq$ $-\beta_{3}\left(\|\eta\|^{2}+\|\tilde{\chi}\|^{2}\right)$ for some $\beta_{3}>0$, and the result of the theorem now follows from application of the comparison lemma [21, Lemma 3.4].

Remark 3: Our stability results require the tuning parameters $k_{P}$ and $\theta$ to be chosen sufficiently high. By inspecting the details of the proof, it is even possible to construct explicit lower bounds on these parameters. However, these bounds are likely to be conservative and not suitable for practical gain selection. Instead, careful tuning based on trial and error is normally needed for practical implementation.

\section{EXTENSIONS AND ModifiCATIONS}

There are alternative ways to construct the vectors $v_{1}^{\mathrm{b}}$ and $v_{2}^{\mathrm{b}}$ on the basis of $f^{\mathrm{b}}$ and $m^{\mathrm{b}}$, with corresponding reference vectors based on $\operatorname{sat}_{M_{f}}\left(\hat{f}^{\mathrm{e}}\right)$ and $m^{\mathrm{e}}$. For the experiments presented in Section VI, we choose to define $v_{1}^{\mathrm{b}}$ as a normalized 
version of $f^{\mathrm{b}}$, and $v_{2}$ as a normalized version of $f^{\mathrm{b}} \times m^{\mathrm{b}}$. Corresponding reference vectors $\hat{v}_{1}^{\mathrm{e}}$ and $\hat{v}_{2}^{\mathrm{e}}$ are defined as normalized versions of $\operatorname{sat}_{M_{f}}\left(\hat{f}^{\mathrm{e}}\right)$ and $\operatorname{sat}_{M_{f}}\left(\hat{f}^{\mathrm{e}}\right) \times m^{\mathrm{e}}$. It can be confirmed from the above proof that the theoretical results still hold, as long as $\hat{\sigma}$ is uniformly bounded and Lipschitz continuous with respect to $\operatorname{sat}_{M_{f}}\left(\hat{f}^{\mathrm{e}}\right)$.

As mentioned in Section II, our representation of PVA is not necessarily the best when it comes to using the estimates for control or for displaying them to a user. However, the estimates can easily be translated to a more practical format. In the observer presented in Section VI, we translate the position estimate $\hat{p}^{\mathrm{e}}$ into estimates $\hat{\mu}, \hat{l}$, and $\hat{h}$ of latitude, longitude, and height with respect to the WGS-84 reference ellipsoid. Such a transformation is typically performed using an iterative procedure, such as that of Hofmann-Wellenhof, Lichtenegger, and Collins [22]. To avoid significant computational overhead, we perform only one iteration of this algorithm at each time step of the discretized algorithm, while carrying the computed latitude over to the next step as an initial condition. Based on the computed latitude and longitude, we compute a quaternion $\hat{q}_{\mathrm{e}}^{\mathrm{n}}=\hat{q}_{\mu} \otimes \hat{q}_{l}$, where $\hat{q}_{l}=[\cos (\hat{l} / 2) ; 0 ; 0 ;-\sin (\hat{l} / 2)]$ and $\hat{q}_{\mu}=$ $[\cos ((\hat{\mu}+\pi / 2) / 2) ; 0 ; \sin ((\hat{\mu}+\pi / 2) / 2) ; 0]$, representing the rotation between the ECEF and NED coordinate systems. This quaternion is used to transform $\hat{v}^{\mathrm{e}}$ into a more convenient estimate $\hat{v}^{\mathrm{n}}=R\left(\hat{q}_{\mathrm{e}}^{\mathrm{n}}\right) \hat{v}^{\mathrm{e}}$ in NED, and to compute a more natural attitude estimate $\hat{q}_{\mathrm{b}}^{\mathrm{n}}=\hat{q}_{\mathrm{e}}^{\mathrm{n}} \otimes \hat{q}_{\mathrm{b}}^{\mathrm{e}}$, representing the rotation between BODY and NED.

\section{EXPERIMENTAL RESULTS}

In this section, we present experimental results based on data gathered with a Piper Cherokee 140 light fixed-wing aircraft, shown in Fig. 1.

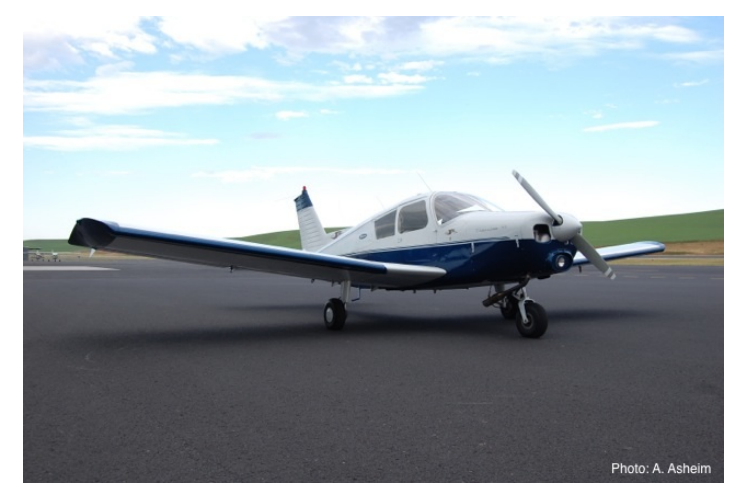

Fig. 1. Aircraft used for data collection

\section{A. Setup and Implementation}

The aircraft is equipped with an Xsens MTi inertial measurement unit, mounted on a bulkhead within the tail, as well as a Mediatek-3329 GPS module with a built-in patch antenna, mounted on top of the instrument panel. The Xsens MTi contains moderately accurate accelerometers, gyros, and magnetometers, with an in-run gyro bias stability of $20 \mathrm{deg} / \mathrm{h}$ $(1 \sigma)$. The measurements are provided at a rate of $100 \mathrm{~Hz}$.
The Mediatek GPS receiver reports position estimates, as well as low-quality speed and heading estimates, at a rate of $10 \mathrm{~Hz}$. All the sensor readings are filtered using thirdorder low-pass filters with a cutoff frequency of $5 \mathrm{~Hz}$, and the GPS readings are converted to $100 \mathrm{~Hz}$ before being provided to the observer. The magnetometer measurements are also filtered with a fourth-order notch filter to remove the predominant component of a square disturbance with period $1.2 \mathrm{~s}$, imposed by the aircraft's anti-collision light.

The observer equations are implemented at $100 \mathrm{~Hz}$ using a forward-Euler discretization. A correction is applied to the attitude estimate to prevent the magnitude of the quaternion from drifting away from unity. Only the position information from the GPS is used in the observer; the speed and heading are discarded. Thus, the matrix $C_{v}$ in the above analysis is empty. The gravity vector $g^{\mathrm{e}}\left(\hat{p}^{\mathrm{e}}\right)$ is modeled by the $J_{2}$ gravity model (see [23]). The chosen tuning parameters are $k_{1}=1$, $k_{2}=1.5, k_{I}=0.008, K_{p p}=0.6 I, K_{v p}=0.11 I, K_{\xi_{p}}=0.006 I$, and $\theta=2$, except for the first $60 \mathrm{~s}$ after initialization, where we use $k_{1}=20, k_{2}=30$, and $k_{I}=0.01$ in order to ensure faster convergence. A parameter projection ensures that $\left\|\hat{b}^{\mathrm{b}}\right\| \leq 0.51 \mathrm{deg} / \mathrm{s}$.

A reference for the estimates is provided by an EKF that takes its measurements from a set of higher-quality sensors, specifically, an ADIS 16488 "tactical grade" IMU, mounted within the cockpit behind the pilot's seat, and a uBlox LEA-6H GNSS receiver with an active antenna mounted on the instrument panel. The ADIS 16488 contains relatively accurate accelerometers, gyros, and magnetometers, with an in-run gyro bias stability of $6.25 \mathrm{deg} / \mathrm{hr}(1 \sigma)$. Measurements are gathered at a rate of $410 \mathrm{~Hz}$. The uBlox GNSS receiver provides position and Doppler-based velocity estimates at a rate of $5 \mathrm{~Hz}$. Accelerometer, gyro, and GNSS position and velocity readings are provided to the EKF after filtering with a third-order, 5-Hz low-pass filter and conversion to $100 \mathrm{~Hz}$. Magnetometer readings are provided by the Xsens MTi instead of the ADIS16488, due to the difficulty of accurately calibrating magnetometers installed in the cockpit. The EKF is implemented as an indirect filter with resetting, but with both prediction and update taking place at $100 \mathrm{~Hz}$. In addition to PVA and gyro bias, the EKF also estimates accelerometer bias. The attitude is represented by a quaternion in the navigation equations, whereas the attitude error in the EKF is represented using a 3-DOF Gibbs vector. The attitude quaternion is reset in the style of a multiplicative EKF (see, e.g., [24]).

\section{B. Results}

We present results from a flight lasting approximately 40 minutes from takeoff to landing. We start by looking in detail at the traffic pattern and landing at the end of the flight, when all the estimates have had ample time to converge. Fig. 2 shows the estimates of the latitude, longitude, and height above the WGS-84 ellipsoid, together with the EKF-generated reference. The discrepancy between the estimated height and the reference is attributable to a systematic difference between the positions reported by the uBlox GNSS receiver 

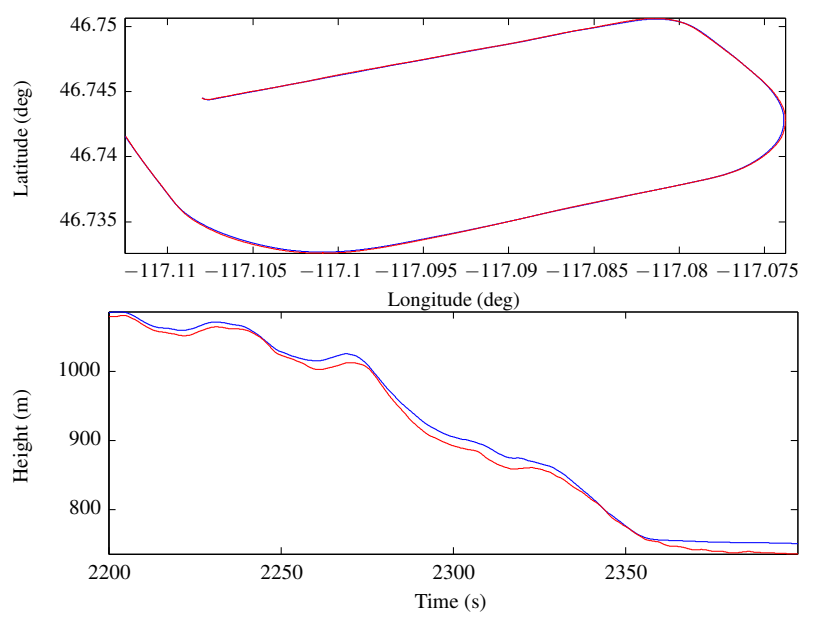

Fig. 2. Position during traffic pattern and landing (blue: reference; red: observer estimate)
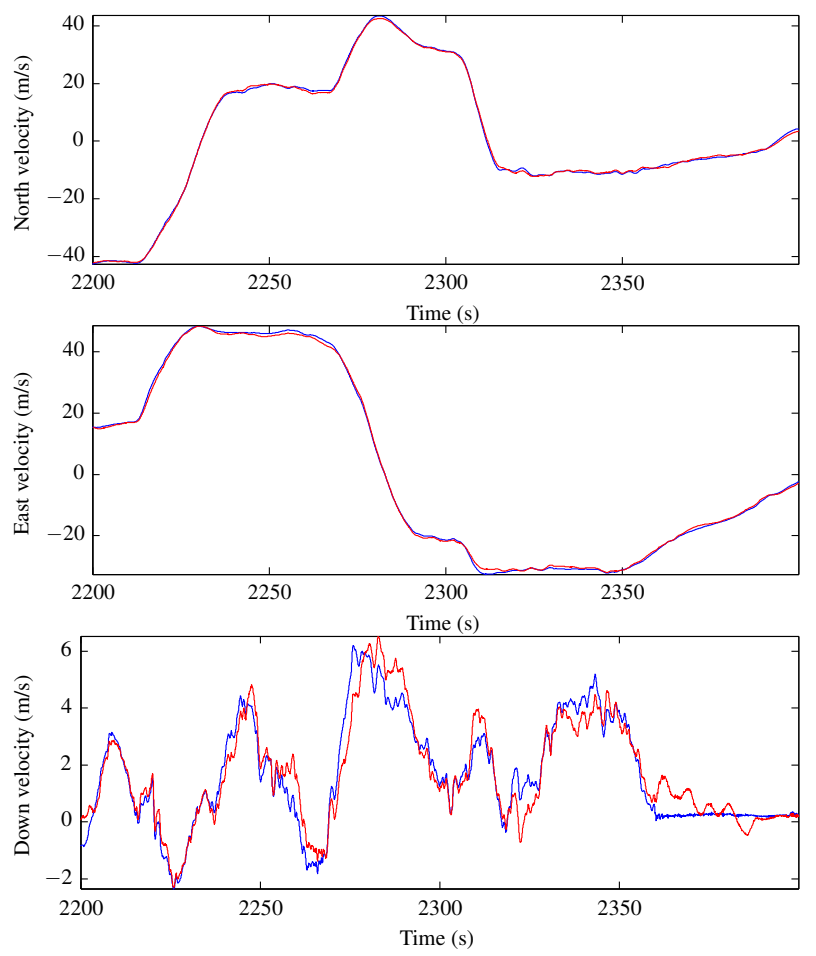

Fig. 3. Velocity during traffic pattern and landing (blue: reference; red: observer estimate)

and the Mediatek GPS module, which is most significant in the vertical direction. Fig. 3 shows the velocity in NED coordinates, and Fig. 4 shows the attitude represented as Euler angles. Although the lower quality of the observer estimates is evident in some places, the agreement with the reference trajectory is generally good.

Next, we look at errors in the observer estimates, relative to the reference, for the entire flight. Fig. 5 shows the NED velocity errors. After an initial transient, the errors mostly remain within a region of approximately $\pm 1 \mathrm{~m} / \mathrm{s}$; however, large spikes can be observed in two places. These
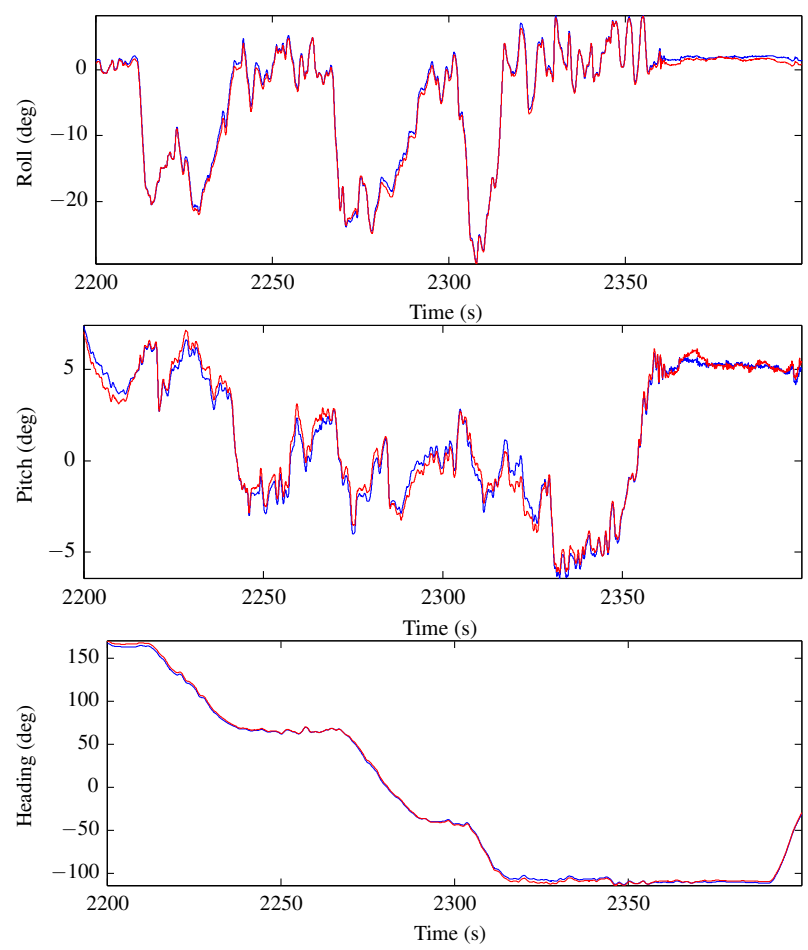

Fig. 4. Attitude during traffic pattern and landing (blue: reference; red: observer estimate)

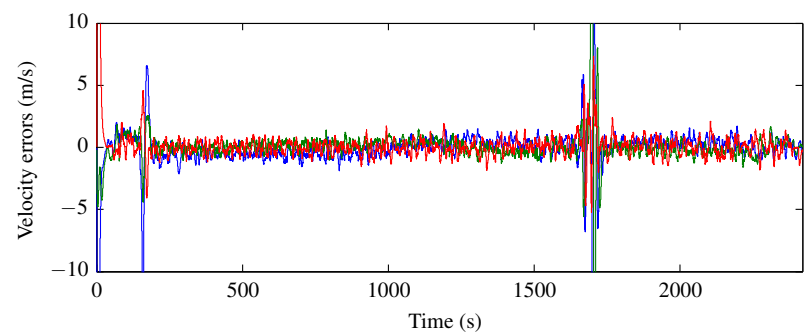

Fig. 5. Velocity error (blue: north; green: east; red: down)

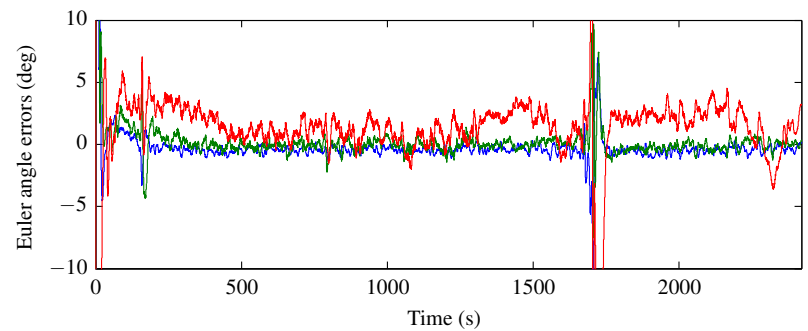

Fig. 6. Attitude error (blue: roll; green: pitch; red: heading)

spikes are due to a significant deterioration in the position reported by the Mediatek GPS module, which is also reflected in spiking HDOP values. Clearly, the observer as currently tuned exhibits an undesirable sensitivity to such deterioration. Most likely, this situation can be improved by detuning the observer during periods of poor GPS quality; however, this is beyond the scope of the current paper. Fig. 6 shows the attitude errors for the entire flight, which 


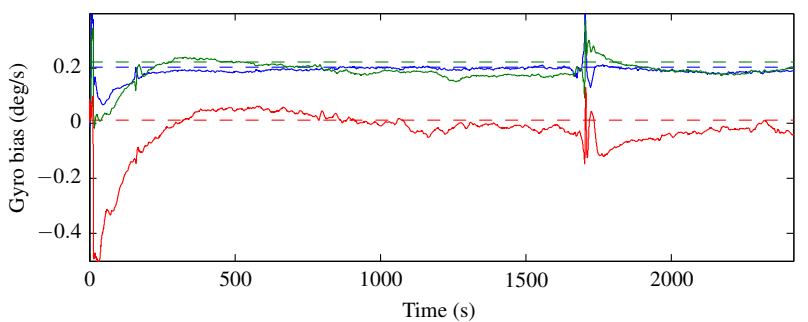

Fig. 7. Gyro bias estimate (solid) and bias computed at standstill (dashed) (blue: roll; green: pitch; red: yaw)

exhibit similar spikes. Except for these spikes, the roll and pitch errors remain mostly in the region $\pm 1 \mathrm{deg}$, but the heading error tends to be somewhat larger. The reason for the larger heading error is not precisely known; however, it is likely related to uncalibrated magnetic disturbances. These disturbances also affect the heading reference, thus making it less reliable.

We end by showing a plot of the estimated gyro bias for the entire flight, compared to a reference bias obtained at standstill after the flight. Again, spikes due to deteriorating GPS quality are evident; however, the close relationship between the estimated bias and the standstill reference is obvious.

\section{CONCluding Remarks}

In this paper we have presented a semiglobally stable nonlinear observer for GNSS/INS integration, where the attitude is represented by a unit quaternion. Experimental results, based on data gathered using a light fixed-wing aircraft, show that the approach has promise, although it is currently sensitive to deterioration in the quality in the GNSS measurements. Increased robustness against this type of error is an interesting topic for future work. Also of interest is the estimation of accelerometer bias, which could be based on previous work by the authors [12], [13].

\section{REFERENCES}

[1] T. I. Fossen, Handbook of Marine Craft Hydrodynamics and Motion Control. Wiley, 2011.

[2] E. Bekir, Introduction to Modern Navigation. World Scientific, 2007.

[3] P. S. Maybeck, Stochastic Models, Estimation, and Control, Volume 1, ser. Mathematics in Science and Engineering. New York: Academic Press, 1979, vol. 141.

[4] R. Phillips and G. Schmidt, "GPS/INS integration," AGARD Lecture Series: System Implications and Innovative Applications of Satellite Navigation, vol. 207, pp. 9.1-9.18, 1996.
[5] M. S. Grewal, L. R. Weill, and A. P. Andrews, Global Positioning Systems, Inertial Navigation, and Integration. New York: Wiley, 2001.

[6] S. Salcudean, "A globally convergent angular velocity observer for rigid body motion," IEEE Trans. Automat. Contr., vol. 36, no. 12, pp. 1493-1497, 1991.

[7] J. Thienel and R. M. Sanner, "A coupled nonlinear spacecraft attitude controller and observer with an unknown constant gyro bias and gyro noise," IEEE Trans. Automat. Contr., vol. 48, no. 11, pp. 2011-2015, 2003.

[8] R. Mahony, T. Hamel, and J.-M. Pflimlin, "Nonlinear complementary filters on the Special Orthogonal Group," IEEE Trans. Automat. Contr., vol. 53 , no. 5, pp. $1203-1218,2008$.

[9] R. Mahony, T. Hamel, J. Trumpf, and C. Lageman, "Nonlinear observers on $\mathrm{SO}(3)$ for complementary and compatible measurements: A theoretical study," in Proc. IEEE Conf. Dec. Contr./Chinese Contr. Conf., Shanghai, China, 2009, pp. 6407-6412.

[10] P. Batista, C. Silvestre, and P. J. Oliveira, "GES attitude observers - Part I: Multiple general vector observations," in Proc. IFAC World Congr., Milan, Italy, 2011, pp. 2985-2990.

[11] — "GES attitude observers - Part II: Single vector observations," in Proc. IFAC World Congr., Milan, Italy, 2011, pp. 2991-2996.

[12] H. F. Grip, T. I. Fossen, T. A. Johansen, and A. Saberi, "Attitude estimation based on time-varying reference vectors with biased gyro and vector measurements," in Proc. IFAC World Congr., Milan, Italy, 2011, pp. 8497-8502.

[13] _ _ "Attitude estimation using biased gyro and vector measurements with time-varying reference vectors," IEEE Trans. Automat. Contr. vol. 57 , no. 5, pp. $1332-1338,2012$.

[14] J. L. Crassidis, F. L. Markley, and Y. Cheng, "Survey of nonlinear attitude estimation methods," J. Guid. Contr. Dynam., vol. 30, no. 1, pp. 12-28, 2007.

[15] B. Vik and T. I. Fossen, "A nonlinear observer for GPS and INS integration," in Proc. IEEE Conf. Dec. Contr., Orlando, FL, 2001, pp. 2956-2961.

[16] M.-D. Hua, "Attitude estimation for accelerated vehicles using GPS/INS measurements," Contr. Eng. Pract., vol. 18, no. 7, pp. 723732, 2010.

[17] T. Hamel and R. Mahony, "Attitude estimation on $\mathrm{SO}(3)$ based on direct inertial measurements," in Proc. IEEE Int. Conf. Robotics Automation, Orlando, FL, 2006, pp. 2170-2175.

[18] H. F. Grip, A. Saberi, and T. A. Johansen, "Observers for cascaded nonlinear and linear systems," in Proc. IEEE Conf. Dec. Contr., Orlando, FL, 2011, pp. 3331-3337.

[19] - " "Observers for interconnected nonlinear and linear systems," Automatica, vol. 48, no. 7, pp. 1339-1346, 2012.

[20] H. F. Grip, T. I. Fossen, T. A. Johansen, and A. Saberi, "A nonlinear observer for integration of GNSS and IMU measurements with gyro bias estimation," in Proc. American Contr. Conf., Montreal, Canada, 2012, pp. 4607-4612.

[21] H. K. Khalil, Nonlinear Systems, 3rd ed. Upper Saddle River, NJ: Prentice-Hall, 2002.

[22] B. Hofmann-Wellenhof, H. Lichtenegger, and J. Collins, "Global Positioning System: Theory and practice," 1994.

[23] D. Y. Hsu, "Comparison of four gravity models," in Proc. Pos. Loc. Nav. Symp., 1996, pp. 631-635.

[24] F. L. Markley, "Attitude error representations for Kalman filtering," $J$. Guid. Contr. Dynam., vol. 26, no. 2, pp. 311-317, 2003. 